\title{
Extracorporeal membrane oxygenation as a bridge to pulmonary transplantation
}

\author{
Charles W. Hoopes, MD, ${ }^{\mathrm{a}}$ Jasleen Kukreja, MD, ${ }^{\mathrm{b}}$ Jeffery Golden, MD, ${ }^{\mathrm{c}}$ Daniel L. Davenport, PhD, ${ }^{\mathrm{a}}$ \\ Enrique Diaz-Guzman, MD, ${ }^{\mathrm{d}}$ and Joseph B. Zwischenberger, $\mathrm{MD}^{\mathrm{a}}$
}

\begin{abstract}
Objective: Acute clinical deterioration preceding death is a common observation in patients with advanced interstitial lung disease and secondary pulmonary hypertension. Patients with pulmonary arterial hypertension refractory to medical therapy are also at risk of sudden cardiac death (cor pulmonale). The treatment of these patients remains complex, and the findings from retrospective studies have suggested that intubation and mechanical ventilation are inappropriate given the universally poor outcomes. Extracorporeal support technologies have received limited attention because of the presumed inability to either recover cardiopulmonary function in the patient with end-stage disease or the presumed inability to proceed to definitive therapy with transplantation.
\end{abstract}

\begin{abstract}
Methods: A retrospective review was performed of 31 patients from 2 institutions placed on extracorporeal membrane oxygenation as a bridge to lung transplantation compared with similar patients without extracorporeal membrane oxygenation at the same institutions and comparison groups queried from the United Network for Organ Sharing database.
\end{abstract}

Results: We have transplanted 31 patients with refractory lung disease from mechanical artificial lung support. Of the 31 patients, 19 were ambulatory at transplantation. Pulmonary fibrosis $(42 \%)$, cystic fibrosis $(20 \%)$, and pulmonary hypertension (16\%) were the most common diagnostic codes and acute cor pulmonale (48\%) and hypoxia (39\%) were the most common indications for device deployment. The average duration of extracorporeal membrane oxygenation support was 13.7 days (range, 2-53 days), and the mean survival of all patients bridged to pulmonary transplantation was 26 months (range, 54 days to 95 months). The 1-, 3-, and 5-year survival was $93 \%, 80 \%$, and 66\%, respectively. The duration of in-house postoperative transplant care ranged from 12 to 86 days (mean, 31 days). Patients requiring an extracorporeal membrane oxygenation bridge had comparable survival to that of the high acuity patients transplanted without extracorporeal membrane oxygenation support in the Scientific Registry of Transplant Recipients database but were at a survival disadvantage compared with the high-acuity patients (lung allocation score, $>50)$ transplanted at the same center who did not require mechanical support $(P<.001)$.

Conclusions: These observations challenge current assumptions about the treatment of selected patients with end-stage lung disease and suggest that "salvage transplant" is both technically feasible and logistically viable. Widespread adoption of artificial lung technology in lung transplant will require the design of clinical trials that establish the most effective circumstances in which to use these technologies. A discussion of a clinical trial and reconsideration of current allocation policy is warranted. (J Thorac Cardiovasc Surg 2013;145:862-8)

Implementation of the lung allocation scoring (LAS) system has decreased the overall waitlist mortality for patients needing pulmonary transplantation. ${ }^{1}$ However, specific disease categories-pulmonary fibrosis with secondary

From the Departments of Surgery ${ }^{\mathrm{a}}$ and Pulmonary Medicine, ${ }^{\mathrm{d}}$ University of Kentucky College of Medicine, Lexington, Ky; and the Departments of Surgery ${ }^{\mathrm{b}}$ and Pulmonary Medicine, ${ }^{\mathrm{c}}$ University of California, San Francisco, School of Medicine, San Francisco, Calif.

Disclosures: Authors have nothing to disclose with regard to commercial support.

Read at the 38th Annual Meeting of The Western Thoracic Surgical Association, Maui, Hawaii, June 27-30, 2012.

Received for publication July 11, 2012; revisions received Dec 7, 2012; accepted for publication Dec 7, 2012; available ahead of print Jan 14, 2013.

Address for reprints: Charles W. Hoopes, MD, Department of Surgery, University of Kentucky College of Medicine, 750 South Limestone, A301, Lexington, KY 40536-0284 (E-mail: charles.hoopes@uky.edu).

$0022-5223 / \$ 36.00$

Copyright (c) 2013 by The American Association for Thoracic Surgery http://dx.doi.org/10.1016/j.jtcvs.2012.12.022 pulmonary hypertension and medically refractory pulmonary arterial hypertension-remain at high risk of acute clinical decompensation and have not been afforded the same waitlist survival advantage. ${ }^{2}$ Mechanical ventilation is not an effective management strategy for patients with end-stage lung disease awaiting transplantation ${ }^{3}$ and "instituting assisted ventilation in patients with predominantly fibrotic forms of interstitial lung disease is of questionable value, perhaps even futile." 4 We describe our experience with extracorporeal technologies (extracorporeal membrane oxygenation [ECMO]) as a bridge to transplantation in patients with fulminant respiratory failure and present an institutional algorithm for the treatment of "transplant eligible" patients with acute respiratory syndromes.

\section{METHODS}

We performed a retrospective review of prospectively collected data of all patients undergoing ECMO as a bridge to lung transplantation from 


\section{Abbreviations and Acronyms \\ BOS = bronchiolitis obliterans syndrome \\ ECMO $=$ extracorporeal membrane oxygenation \\ LAS = lung allocation score \\ PEA $=$ pulseless electrical activity \\ PECLA $=$ pumpless extracorporeal lung assist \\ $\mathrm{RV} \quad=$ right ventricular \\ UNOS = United Network for Organ Sharing \\ $\mathrm{VA}=$ venoarterial}

January 2003 to June 2012 at 2 different institutions (University of California, San Francisco, School of Medicine and University of Kentucky College of Medicine). All patients followed an ECMO deployment algorithm managed by a single surgeon (Figure 1).

We compared the survival of these patients (ECMO group) with that of other lung transplant patients from our institutions with LAS scores greater than 50 (LAS score $>50$ group) and 2 other patient groups queried from the United Network for Organ Sharing (UNOS) database. The UNOS groups included isolated double lung transplants performed from May 2005 to June 2011 with a transplantation diagnosis of cystic fibrosis, idiopathic pulmonary fibrosis, or pulmonary arterial hypertension and a LAS score greater than 50: without ECMO (UNOS LAS score $>50$ group) and with ECMO (UNOS ECMO group) at the time of matching. The 3 -year survival was compared across the 4 groups using the Kaplan-Meier method and the log-rank test. Statistical analyses were performed using SPSS, version 19.0, software (SPSS, Chicago, Ill).

\section{ECMO Deployment}

An algorithm for ECMO deployment is shown in Figure 1. Using this algorithm, all mechanically ventilated patients with hemodynamic instability and/or acute respiratory failure undergo open femoral cannulation for stabilization using venoarterial ECMO (femoral arterial cannula and a long venous cannula deployed to the right atrium). Patients are weaned from paralytics, sedation, and vasopressor support. We deployed an $8 \mathrm{~F}$ distal femoral arterial flow cannula if clinically indicated to preclude limb ische$\mathrm{mia}$; however, this is rarely necessary during the initial deployment $(30 \%$ incidence). We do not consider femoral venoarterial (VA) ECMO an appropriate method of long-term support, because it precludes ambulatory status. In awake patients with nonfocal neurologic examination findings and adequate end organ perfusion, the VA ECMO blood path is reversed and the centrifugal pump excluded from the circuit-arteriovenous pumpless extracorporeal lung assist (PECLA). Native cardiac output generates flows of 1.6 to $2.2 \mathrm{~L} / \mathrm{min}$ from the femoral arterial cannula through the Quadrox oxygenator, with return to the right atrium. Patients who remain hemodynamically stable — even with low oxygen saturation—can be converted to venovenous ECMO with a dual lumen catheter after 30 minutes of femoral PECLA support. Echocardiographic evaluation during PECLA support has proved a useful adjunct in assessing global ventricular function and evidence of cor pulmonale (right ventricular [RV] to left ventricular ratio in diastole, quantification of tricuspid valvular insufficiency, and inadequate left ventricular preload). Patients with ongoing hemodynamic requirements (RV failure/pulmonary hypertension) are converted to central cannulation (right atrium [RA] to pulmonary artery [PA], PA to left atrium, RA to ascending aorta) with off-label use of available ventricular assist device cannulas (Thoratec PVAD, Pleasanton, Calif; Abiomed AB5000, Danvers, Mass). Cannulation is by median sternotomy or bilateral anterolateral thoracotomy, depending on the subsequent planned operation (eg, heart-lung, bilateral lung) and the acuity of deployment.

"Hybrid ECMO" with venovenous duel lumen cannula support and an associated atrial septostomy ${ }^{5}$ has proved useful in patients who require mechanical support and are not considered candidates for central cannulation. In our experience, induction anesthesia and mechanical ventilation precipitate cardiovascular collapse (pulseless electrical activity [PEA]) in nearly one half of the patients presenting with end-stage RV failure related to pulmonary arterial hypertension. These patients are placed directly on VA ECMO under local anesthesia. Venoarteriovenous ECMO with a dual lumen venovenous cannula and simultaneous postoxygenator blood return to both the right atrium and right subclavian artery has also proved an effective mode of support in ambulatory patients. ${ }^{6}$

All patients receive continuous intravenous heparin with a target activated clotting time of 140 to 180 seconds. We use a QUADROX oxygenator and centrifugal pump (Thoratec CentriMag) for long-term extracorporeal support ( $>72$ hours). No protocols have defined the absolute criteria for transfusion or laboratory analysis, and one half the patients received transfusions before transplantation. All patients mechanically ventilated at ECMO support underwent tracheostomy. All donor lungs fulfilled standard criteria. All transplants were performed with cardiopulmonary bypass. Any neurologic deficit was a contraindication to continued ECMO bridge support.

\section{Ambulatory ECMO Approach}

Starting in 2005, all ECMO-supported patients were subject to an ambulatory management program. All patients were weaned off sedation and were awake after ECMO deployment. The patients were evaluated by a multidisciplinary team involving physical and occupational therapy, respiratory therapy, speech pathology, and a nutritionist. Once hemodynamic stability was achieved (weaned off vasopressors), the patients were assisted by the multidisciplinary team and began ambulation trials with a goal to ambulate more than $200 \mathrm{ft}$ before transplantation. A perfusion specialist was available while the patients ambulated to assist the nursing personnel with the ECMO circuit. Physical therapy also provided range of motion and weight lifting limb exercises to improve upper and lower extremity strength. The patients were allowed to eat by mouth if the swallow evaluation findings were normal.

\section{RESULTS}

From January 2003 to June 2012, 31 patients were bridged to lung transplantation from an "ECMO bridge." Patient age and gender, transplant date, diagnosis, indication for ECMO, duration of support (days), cannulation strategy, operation, and outcomes are listed in Table 1. Of the 31 patients, 18 were ambulatory at transplantation. The etiology of the postoperative deaths included noncompliance in retransplanted cystic fibrosis (patient 1, recurrent multidrug-resistant pseudomonas pneumonia), bronchiolitis obliterans syndrome (BOS) beyond 5 years with renal dysfunction precluding repeat transplantation (patient 2), BOS at 34 months with performance status precluding repeat transplantation (patient 4), multiorgan system failure at 10 months in an outside hospital patient (patient 12), metastatic adenocarcinoma with an unknown primary at 13 months (patient 13), and technical misadventure (catastrophic air embolus/patent foramen ovale, patient 21). Three patients (patients 9, 23, and 24) were not receiving mechanical ventilation at ECMO cannulation. Patient 5 (with acute respiratory distress syndrome) underwent repeat transplantation from venovenous duel lumen cannula ECMO (University of Iowa) in February 2012 (BOS/hypoxia). One patient continued with ECMO after implantation 


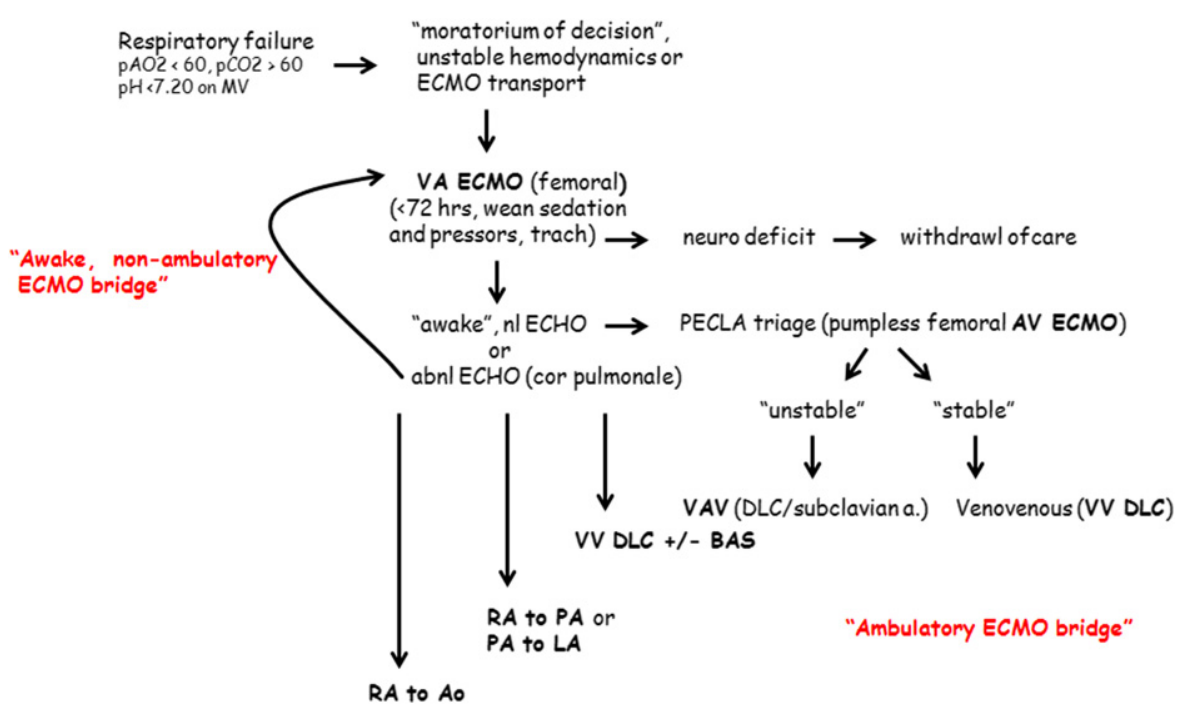

FIGURE 1. Management algorithm for potential bridge to transplant candidates. All hemodynamically unstable patients with respiratory or cardiopulmonary failure and patients requiring transport were stabilized with femoral venoarterial (VA) extracorporeal membrane oxygenation (ECMO). Within 72 hours of deployment, patients who are awake ("neuro intact") and without inotropic requirement have the ECMO blood path reversed (femoral arteriovenous [AV] ECMO) without centrifugal pump support (pumpless extracorporeal lung assist [PECLA]). Patients tolerating PECLA were converted to venovenous (VV) dual lumen cannula $(D L C)$ support in preparation for ambulation. Patients with ongoing need for inotropic or centrifugal pump support were converted to central cannulation or hybrid venoarteriovenous $(V A V)$ ECMO in preparation for ambulation. $p A O 2$, Arterial partial pressure of oxygen; $p C O 2$, partial pressure of carbon dioxide; $M V$, mechanical ventilation; $h r s$, hours; trach, tracheostomy; neuro, neurologic; $n l$, normal; ECHO, echocardiogram; abnl, abnormal; $B A S$, balloon atrial septostomy; $R A$, right atrium; $P A$, pulmonary artery; $L A$, left atrium; Ao, ascending aorta.

for primary graft dysfunction (patient 4 , central cannulation RA to ascending aorta for 3 days). Excluding the perioperative death (patient 21), all patients were discharged from the hospital. The duration of in-house postoperative transplant care ranged from 12 to 86 days (mean, 31 days).

Three patients had significant complications of device deployment. Patient 8 required reoperation 3 days after ECMO for cardiac tamponade and repositioning of the pulmonary artery outflow graft. Patient 21 underwent a cannula exchange for a fracture of the dual lumen Avalon venovenous ECMO circuit on post-ECMO day 10 - this was unrelated to the subsequent perioperative death. Patient 18, a 21-year-old woman with pulmonary veno-occlusive disease who underwent 28 days of femoral VA ECMO for cardiopulmonary collapse, was unable to ambulate after an unremarkable bilateral lung transplant. Subsequent magnetic resonance imaging findings were consistent with "watershed" ischemia of the proximal thoracic spinal cord. We considered this a complication of prolonged retrograde perfusion common to the femoral VA ECMO blood path but are unaware of any previous reports of a similar complication. The patient regained normal ambulatory status but the requirements of neurologic rehabilitation accounted for the longest period of post-transplant care within the case series (86 days).

The 1- and 3-year actuarial survival for the ECMO group was $93 \%$ and $83 \%$, respectively (Figure 2). This was significantly worse than the survival for similar patients transplanted without mechanical support (LAS score $>50$ group, $P<.001)$. Approximately $30 \%$ of the patients transplanted without ECMO had been hospitalized before transplantation, and the outcomes of the LAS score $>50$ group were statistically better than predicted $(P<.05$; www.SRTR.org). Survival of the "ECMO bridge" patients was statistically significantly better than that of the 2 UNOS comparison groups (UNOS LAS score $>50$ and UNOS ECMO; $P<.001)$.

\section{DISCUSSION}

The present case series had the limitations common to all retrospective data analyses, and it is difficult to make legitimate comparisons among the recent reports of ECMO as a bridge to transplantation. Regional differences in organ allocation policy, institutional differences in the logistic design and deployment criteria of extracorporeal circuits, and surgeon-specific preferences regarding organ selection and operative technique can have a profound effect on the outcomes. Nonetheless, in the present case series, the survival of patients "ECMO bridged" to transplant was $97 \%$ at 30 days. The 1 -year outcomes of the patients transplanted from an ECMO bridge exceeded $90 \%$, the 3 -year outcomes exceeded $80 \%$, and 3 patients have survived beyond 5 years $(66 \%)-1$ of whom underwent repeat transplantation from a second ECMO bridge for BOS 68 months after his original implant date. However, although the survival data within the present case series were comparable or superior to those previously reported, ${ }^{7,8}$ high acuity patients (LAS 
TABLE 1. Patient demographics

\begin{tabular}{|c|c|c|c|c|c|c|c|}
\hline Pt. no. & Age (y), gender, surgery date & Diagnosis & Indication & $\operatorname{DOS}(\mathrm{d})$ & Cannulation & Procedure & Outcome \\
\hline 1 & $33, \mathrm{M}, 10 / 03$ & $\mathrm{CF} / \mathrm{BOS}$ & Hypoxia & 2 & VA (f) & Repeat BLTx & Death at $14 \mathrm{mo}(\mathrm{NC})$ \\
\hline 2 & $44, \mathrm{M}, 4 / 04$ & IPF & RV/PEA & 5 & VA (f) & BLTx & Death at $61 \mathrm{mo}(\mathrm{BOS})$ \\
\hline 3 & $52, \mathrm{M}, 7 / 04$ & IPF & RV/PEA & 7 & VA (f) & BLTx & \\
\hline 4 & $61, \mathrm{M}, 7 / 05$ & IPF & RV/PEA & $3(3)$ & VA (f), (RA to Ao) & BLTx & Death at 34 mo (BOS) \\
\hline 5 & $28, \mathrm{M}, 6 / 06$ & ARDS & Hypoxia & 3 & VV & BLTx & \\
\hline 6 & $32, \mathrm{~F}, 1 / 08$ & $\mathrm{CF}$ & Hypoxia & 3 & VA (f) & BLTx & \\
\hline 7 & $61, \mathrm{~F}, 3 / 08$ & Scl/ILD & RV/PEA & 6 & VA (f) & BLTx & \\
\hline $8^{*}$ & $58, \mathrm{M}, 1 / 09$ & IPF & Hypoxia/PEA & 11 & RA to Ao & BLTx & \\
\hline $9 *$ & $59, \mathrm{M}, 2 / 09$ & BOS & Hypoxia & 11 & VV (DL) & BLTx & \\
\hline $10^{*}$ & $32, \mathrm{~F}, 2 / 09$ & PPHTN & PEA & 13 & RA to Ao & HLTx & \\
\hline 11 & $15, \mathrm{~F}, 7 / 09$ & PVOD & RV/PEA & 16 & VA (f) & BLTx & \\
\hline $12 *$ & $48, \mathrm{M}, 2 / 10$ & Dm/ILD & RV/PEA & 33 & VA (f), PA to LA & BLTx & Death at $10 \mathrm{mo}$ (MOSF) \\
\hline $13^{*}$ & $64, \mathrm{M}, 3 / 10$ & PHTN & RV/PEA & 20 & PA to LA & BLTx & Death at 13 mo (ACA) \\
\hline $14^{*}$ & $39, \mathrm{M}, 5 / 10$ & $\mathrm{CF} / \mathrm{BOS}$ & Hypoxia & 4 & VV (DL) & Repeat BLTx & \\
\hline $15^{*}$ & $49, \mathrm{M}, 5 / 10$ & Sarcoid & RV/PEA & 14 & RA to Ao & HLTx & \\
\hline $16^{*}$ & $48, \mathrm{M}, 6 / 10$ & AIP & Hypoxia & 24 & VV, RA to PA & BLTx & \\
\hline $17^{*}$ & $32, \mathrm{M}, 6 / 10$ & IPF & IPF & 36 & PA to LA & BLTx & \\
\hline 18 & $21, \mathrm{~F}, 7 / 10$ & PVOD & RV/PHTN & 28 & VA (f) & BLTx & \\
\hline $19 *$ & $25, \mathrm{~F}, 8 / 10$ & $\mathrm{CF} / \mathrm{BPF}$ & Hypercarbia & 14 & VV (DL) & BLTx & \\
\hline $20 *$ & $35, \mathrm{M}, 1 / 11$ & $\mathrm{CF}$ & Hypoxia & 2 & VV (DL) & BLTx & \\
\hline 21 & $51, \mathrm{M}, 3 / 11$ & IPF & Hypoxia & 18 & $\mathrm{VV}$ (DL), VA(f) & BLTx & Death POD1 (neuro) \\
\hline 22 & $40, F, 4 / 11$ & Scl/ILD & Hypoxia & 2 & VA (f) & BLTx & \\
\hline $23^{*}$ & $68, \mathrm{M}, 4 / 11$ & CWP & PHTN (ei) & 3 & VV (DL) & BLTx & \\
\hline $24^{*}$ & $68, \mathrm{~F}, 4 / 11$ & PVOD & Syncope & 2 & VV (DL)/septostomy & BLTx & \\
\hline $25^{*}$ & $23, \mathrm{~F}, 5 / 11$ & $\mathrm{CF}$ & Hypoxia & 15 & VV (DL) & BLTx & \\
\hline 26 & $59, \mathrm{M}, 6 / 11$ & IPF & RV/PEA & 4 & VA (f) & BLTx & \\
\hline $27^{*}$ & $26, \mathrm{M}, 8 / 11$ & ARDS & Hypoxia & 53 & VV (DL) & BLTx & \\
\hline $28^{*}$ & $52, \mathrm{~F}, 12 / 11$ & $\mathrm{CF}$ & Hypoxia & 13 & VAV hybrid & BLTx & \\
\hline $29 *$ & $65, \mathrm{M}, 1 / 12$ & IPF & Hypoxia/RV & 6 & VV (DL) & BLTx & \\
\hline 30 & $47, \mathrm{M}, 1 / 12$ & IPF & Hypoxia/RV & 4 & VA (f) & BLTx & \\
\hline $31^{*}$ & $53, \mathrm{M}, 4 / 12$ & ARDS & Hypoxia & 48 & VV (DL) & BLTx & \\
\hline
\end{tabular}

Pt. no., Patient number; $D O S$, duration of support; $M$, male; $C F$, cystic fibrosis; $B O S$, bronchiolitis obliterans syndrome; $V A(f)$, venoarterial (femoral vein to femoral artery/retrograde); $B L T x$, bilateral lung transplantation; $N C$, nasal cannula; $I P F$, idiopathic pulmonary fibrosis; $R V$, right ventricular; $P E A$, pulseless electrical activity; $R A$ to $A o$, right atrium to ascending aorta ("walking bypass"); $R A$ to $P A$, right atrium to pulmonary artery ("oxyRVAD"); $A R D S$, acute respiratory distress syndrome; $V V$, venovenous extracorporeal membrane oxygenation (internal jugular to femoral vein, femoral vein to internal jugular); $F$, female; $S c l$, scleroderma; $I L D$, interstitial lung disease; $V V(D L)$, venovenous right internal jugular dual lumen cannula; $P P H T N$, persistent pulmonary hypertension; $H L T x$, heart-lung transplantation; $P V O D$, pulmonary veno-occlusive disease; $D m$, dermatomyositis; PA to LA, pulmonary artery to left atrium ("pulmonary bypass"); $M O S F$, multiorgan system failure; $P H T N$ ( $e i$ ), pulmonary hypertension; $A C A$, adenocarcinoma; $A I P$, acute interstitial pneumonitis; $B P F$, bronchopleural fistula; $P O D$, postoperative day; neuro, neurologic; VAV (hybrid), right internal jugular dual lumen catheter with both right subclavian artery and right internal jugular return; $C W P$, coal workers' pneumoconiosis. *Patients ambulatory at transplantation $(\mathrm{n}=18)$.

score $>50$ ) within our institutions who require an ECMO bridge were at a survival disadvantage compared with high acuity patients (LAS score $>50$ ) who did not require mechanical support. In contrast, patients in our case series transplanted from an ECMO bridge were at a survival advantage compared with patients with an LAS score greater than 60 in the national Scientific Registry of Transplant Recipients database (76.7\% 1-year survival; www.SRTR.org). Although the costs were approximately $30 \%$ greater for patients transplanted from an ECMO bridge than for patients transplanted from home, the cost differential was less compared with in-house patients with LAS scores exceeding 50 who did not require mechanical support. Although several "bridged" patients have returned to either partial or fulltime employment, we do not have adequate data to calculate the "cost per life-year saved."
Given the logistic and fiduciary burden, it is worth considering the efficacy of ECMO bridging in pulmonary transplantation. Four patients UNOS listed for transplantation and placed on ECMO for clinical decompensation had withdrawal of care. These included VA femoral cannulation of a 60-year-old man with scleroderma/pulmonary hypertension (after PEA arrest) and evidence of worsening systemic vasculitis, a 47-year-old woman with sarcoidosis who underwent delayed femoral cannulation for cor pulmonale/ PEA arrest and progressed to anoxic brain death (postECMO day 3), a 68-year-old man with idiopathic pulmonary fibrosis and secondary pulmonary hypertension who developed progressive RV failure after deployment of a venovenous dual lumen cannula (Avalon), and a 61-yearold man with interstitial lung disease receiving immunosuppressive therapy who was converted from venovenous duel 


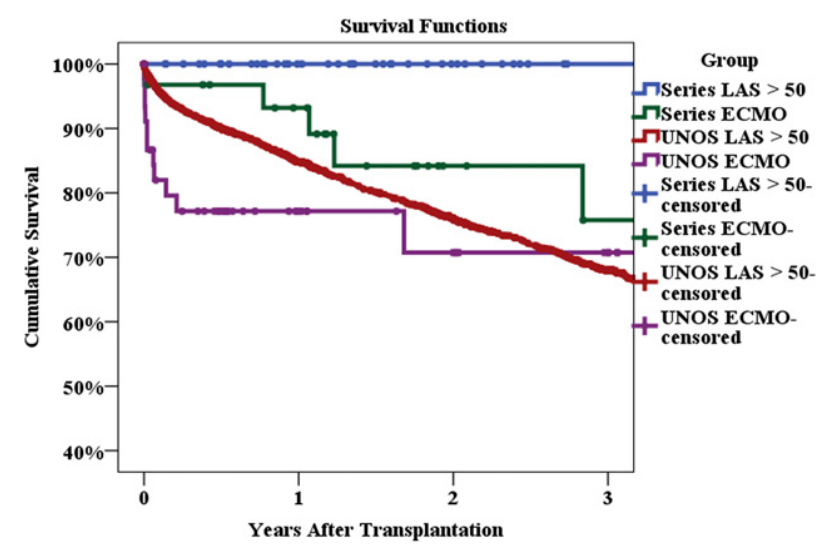

FIGURE 2. Kaplan-Meier 3-year survival for patients transplanted from an extracorporeal membrane oxygenation (ECMO) bridge (Series $\mathrm{ECMO}, \mathrm{n}=31$, green) and patients on our institutional waitlist with lung allocation system (LAS) scores greater than 50 transplanted during the same period (June 2005 through June 2012) without mechanical support (Series LAS score $>50, \mathrm{n}=69$, blue). The institutional outcomes were compared with similar patients from the United Network for Organ Sharing (UNOS) data set transplanted without ECMO support (UNOS LAS score $>50$ group, $\mathrm{n}=2971$, red $)$ and UNOS registry patients supported with ECMO at transplantation (UNOS ECMO group, $\mathrm{n}=47$, pur$p l e)$. Survival varied significantly among the groups (log-rank $P<.001)$.

lumen cannula support to central ECMO (RA to ascending aorta) and subsequently developed multiorgan system failure/fungal sepsis. Overall, the conversion rate from ECMO bridge to transplant for patients currently listed in UNOS at ECMO support is $87 \%(31 / 35)$.

We consider patients with acute respiratory failure syndromes-who are not UNOS listed at initial ECMO support- to be candidates for transplant from an ECMO bridge. This population of "salvage transplants" represents $27 \%$ of the present case series (patients 5, 8, 15, 16, 17, 27, and 31). These patients were all transferred with mechanical ventilation from an outside hospital with fulminant respiratory failure (1 ECMO transport, 2 on ECMO before transfer). Decisions regarding whether patients accepted from an outside institution for ECMO are receiving ECMO as a "bridge to recovery" or "bridge to transplant" is contingent on the clinical course and underlying diagnosis. On the basis of the institutional epidemiology of ECMO/extracorporeal life support from 2005 to 2012 (Figure 3), one half of the potential candidates (7 of 18) in whom pulmonary function failed to recover and were otherwise candidates for transplantation have received allografts $(40 \%$ conversion rate). This observation should inform subsequent discussions as to whether the "ECMO bridge" is an evolving standard of care ${ }^{9}$ or a specialty practice limited to regional referral centers. ${ }^{10}$ An "awake" patient who does not meet the viability criteria for subsequent transplantation presents a significant logistic and ethical critical care dilemma.

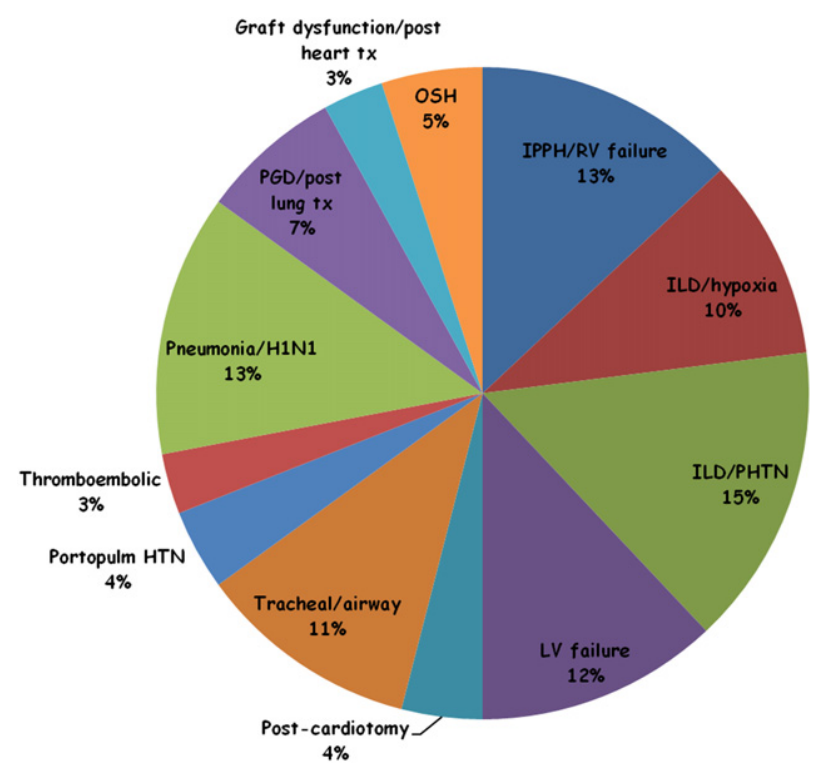

FIGURE 3. Institutional epidemiology of 100 sequential extracorporeal life support deployments (86 patients), single-surgeon experience (2005 to June 2012). Approximately $30 \%$ of extracorporeal membrane oxygenation (ECMO) deployments were for respiratory failure in non-United Network for Organ Sharing waitlisted patients potentially qualifying for pulmonary transplant by disease diagnosis, age, and known associated comorbidities $(\mathrm{n}=26)$. Of these, 8 recovered ("bridge to recovery"). Of the remaining 18 "salvage ECMO" patients, 7 were listed for transplantation and received allografts. The remaining 11 patients had withdrawal of support in the absence of viable transplant candidacy. $t x$, Transplantation; $O S H$, outside hospital; $R V$, right ventricular; $I L D$, interstitial lung disease; $P H T N$, pulmonary hypertension; $L V$, left ventricular; $H T N$, hypertension; $H 1 N 1$, H1N1 influenza; $P G D$, primary graft dysfunction.

The emphasis of contemporary reports on patient "bridging and improvement" 11 and the "support for ambulatory patients without mechanical ventilation"12,13 is consistent with our experience during the past decade. Since January 2006, all patients have been awake and interactive, and 19 patients were ambulatory at transplantation. We routinely preclude active listing of ECMO-supported patients with UNOS until they reach a "threshold of viability." To that end, the "ECMO bridge to transplant" can provide an effective method of pulmonary allograft resource allocation to the sickest of patients, who are likely to benefit. If ECMO is to become for pulmonary transplantation what ventricular assist devices have become for cardiac transplantation, the parameters of effective device deployment will need to be integrated into the current lung allocation system.

Although all extracorporeal technologies share the generalized risk profile of bleeding and thrombosis, our clinical failures were not device related. Inappropriate patient selection and delayed deployment of appropriate technology have been significant predictors of poor patient outcomes. We do not consider the use of an "ECMO bridge" to pulmonary transplantation an isolated surgical procedure. The 
management algorithm is designed to recognize that the extracorporeal technology that will effectively bridge a patient with cystic fibrosis with hypercapnic respiratory failure is markedly different than the device technology required to support a patient with idiopathic pulmonary fibrosis with cor pulmonale and PEA arrest. The clinical goal is an ambulatory patient without respiratory failure and a risk profile for transplantation significantly less than that at the initiation of initial ECMO support. This clinical thought process mirrors that of current ventricular assist device management paradigms in cardiac transplantation.

The broad application of artificial lung technologies to support patients with advanced lung disease has significant public health and policy implications. Most UNOS waitlisted patients described in the present report underwent emergent cannulation in the context of progressive disease (eg, accelerated decompensation phase of pulmonary fibrosis with secondary pulmonary hypertension and cardiopulmonary collapse). An additional group of patients had parenchymal disease not amenable to traditional support with mechanical ventilation (eg, uncontrolled bronchopleural fistula in cystic fibrosis). Transplantation before decompensation would have precluded the need for mechanical support. As noted in the outcomes analysis, our institutional UNOS waitlisted patients with LAS scores greater than 50 who avoided ECMO support had a significant survival advantage. An analysis of lung transplants performed from May 2006 to December 2006 within UNOS region 5 demonstrated that only $4 \%$ of transplanted patients had LAS scores greater than 70 (unpublished data). LAS scores within the local donor-specific area were predictive, with the waiting time reduced by one half for every 5 points greater than an LAS score of 30 . The estimated wait time for a patient with an LAS score greater than 50 was less than 14 days. Had organ allocation been regionalized rather than local, it is possible that several patients "rescued" with an ECMO bridge might have undergone transplantation before clinical decompensation. A discussion of more "regionalized" (nonlocal) allocation of pulmonary allografts - similar to that currently in place for cardiac allografts-is warranted.

\section{CONCLUSIONS}

The use of extracorporeal technologies to salvage, rehabilitate, and, subsequently, transplant patients with nonrecoverable lung disease is technically doable and logistically feasible. Progression of chronic disease with unpredictable and fulminant decompensation (pulmonary fibrosis and pulmonary hypertension), mechanical complications of an established lung disease (bronchopleural fistula in cystic fibrosis), and respiratory failure syndromes without efficacious medical therapy (pulmonary venoocclusive disease) are the diagnostic categories most likely to demonstrate a survival benefit from the application of extracorporeal mechanical support. Algorithm-directed management of ECMO deployment can improve survival. An informed registry of the relevant parameters predicting successful usage of current technologies is necessary and discussion of an appropriately constructed clinical trial is warranted.

Some of the data used in the present report were from the UNOS database and the participating centers. UNOS did not review the analysis and is not responsible for our conclusions.

\section{References}

1. Russo M, Worku B, Iribarne KN, Hong K, Yang JA, Vigneswaran W, et al. Does lung allocation score maximize survival benefit from lung transplantation? J Thorac Cardiovasc Surg. 2011;141:1270-7.

2. Chen H, Shiboski S, Golden J, Gould M, Hays S, Hoopes C, et al. Impact of the lung allocation score on lung transplantation for pulmonary hypertension. Am J Respir Crit Care Med. 2009;180:468-74.

3. Singer J, Blanc PD, Hoopes C, Golden J, Koff J, Leard L, et al. The impact of pretransplant mechanical ventilation on short- and long-term survival after lung transplantation. Am J Transpl. 2011;11:2197-204.

4. Baydur A. Mechanical ventilation in interstitial lung disease. Chest. 2008;133 1062-3.

5. Bacchetta M, Javidfar J, Sonnet J, Kim H, Zwischenberger J, Wang D. Ease of conversion from venovenous extracorporeal membrane oxygenation to cardiopulmonary bypass and venoarterial extracorporeal membrane oxygenation with a bicaval dual lumen catheter. ASAIO J. 2011;57:283-5.

6. Hoopes C, Gurley JC, Zwischenberger JB, Diaz-Guzman E. Mechanical support for pulmonary veno-occlusive disease: combined atrial septostomy and venovenous extracorporeal membrane oxygenation. Semin Thorac Cardiovasc Surg. 2012;24:232-4.

7. Hämmäinen P, Schersten H, Lemström K, Riise GC, Kukkonen S, Swärd K, et al. Usefulness of extracorporeal membrane oxygenation as a bridge to lung transplantation: a descriptive study. J Heart Lung Transplant. 2011;30:103-7.

8. Bermudez CA, Rocha RV, Zaldonis D, Bhama JK, Crespo MM, Shigemura N, et al. Extracorporeal membrane oxygenation as a bridge to lung transplant: midterm outcomes. Ann Thorac Surg. 2011;92:1226-32.

9. Del Sorbo L, Ranieri VM, Keshavjee S. Extracorporeal membrane oxygenation as "bridge" to lung transplantation: what remains in order to make it standard of care? Am J Respir Crit Care Med. 2012;185:699-701.

10. Haneya A, Philipp A, Mueller T, Lubnow M, Pfeifer M, Zink W, et al. Extracorporeal circulatory systems as a bridge to lung transplantation at remote transplant centers. Ann Thorac Surg. 2011;91:250-5.

11. Lang G, Taghavi S, Aigner C, Rényi-Vámos F, Jaksch P, Augustin V, et al. Primary lung transplantation after bridge with extracorporeal membrane oxygenation: a plea for a shift in our paradigms for indications. Transplantation. 2012;93:729-36.

12. Fuehner T, Kuehn C, Hadem J, Wiesner O, Gottlieb J, Tudorache I, et al. Extracorporeal membrane oxygenation in awake patients as bridge to lung transplantation. Am J Respir Crit Care Med. 2012;185:763-8.

13. Garcia J, Iacono A, Kon ZN, Griffith BP. Ambulatory extracorporeal membrane oxygenation: a new approach for bridge-to-lung transplantation. J Thorac Cardiovasc Surg. 2010;139:e137-9.

\section{Discussion}

Dr Michael S. Mulligan (Seattle, Wash). Thanks, Chuck. That represents a lot of work. Very nice paper, and certainly you have done a lot to push the envelope and advance the field. I have a few questions for you.

The first is that I notice there were 5 nonretransplant patients with cystic fibrosis (CF). When they present hypoxic and in hypercarbic failure, they are intubated and then begin to retain infected secretions. Their time on the ventilator is very poorly tolerated and often quickly progress to sepsis. Conversely, the "crashing idiopathic pulmonary fibrosis (IPF)" patient historically does poorly in long-term outcomes. I was wondering how the patients with 
CF fared and is this something we can do to provide advantages for them going forward?

Dr Hoopes. From our perspective, patients with CF are ideal patients because they are less unstable hemodynamically when they present. For patients with CF, we have deployed ECMO in response to mechanical complications of the disease, primarily complications of uncontrolled bronchopleural fistula when patients could not be adequately ventilated. We have very few absolute exclusions to transplant, but we will not transplant someone who is not ambulatory. In the past 4 years, CF patients with profound hypoxia or hypercarbia secondary to disease burden who have required mechanical ventilation have undergone tracheostomy and transition to venovenous ECMO with the expectation that they all become ambulatory prior to transplantation.

Dr Mulligan. So perhaps you are intervening a bit earlier than some of the other centers. Intervening later seems to be associated with survival outcomes that are not quite as good.

The second question I have for you relates to the LAS scoring. Currently, if you consider all the UNOS data, the use of pretransplant ECMO carries a post-transplant odds ratio for mortality of 7 . Your own institutional data showed a negative effect on outcomes, and, currently in the UNOS bylaws, no LAS adjustment is made for being on ECMO. Given that so many of your patients were listed patients, how did you adjust the LAS score when they went on ECMO?

Dr Hoopes. LAS scores reflect the amount of oxygen and the need for mechanical ventilation as the vast majority of patients were intubated and transitioned from mechanical ventilation to ECMO support. From a philosophical standpoint, I would argue that we are going to ultimately end up the same place we are when we consider our cardiac patients with ventricular assist devices. We will actually start lowering the scores of patients on ECMO and deprioritizing them, not increasing their priority score because they would be more stable and actually reperesent a lower risk profile recipient. As you note, currently ECMO cannot be effectively integrated into the LAS system. Nonetheless, in the majority of patients, life-sustaining extracorporeal life support is inherently unstable and we have listed them as though they were receiving mechanical ventilation.

Dr Mulligan. The last question relates to your call for regional sharing. The benefits to critically ill recipients have been outlined; however, significant issues exist with implementing such strategies. Specifically, one would have to very carefully standardize the listing behavior and practices to avoid "gaming" of the system. One would also have to try to make organ acceptance behaviors fairly uniform. Can you speak to some of these barriers to regional sharing?

Thank you very much. Excellent paper.

Dr Hoopes. We are in total agreement. My frustration with transplantation is that it is not currently studied as a public health issue. It is currently viewed as "my list versus your list," and I do not think, under the current system, it would be possible to actually make an LAS system that takes into account regionalization versus local program interests. What I would advocate for in the long run is regionalization of transplantation as a public health resource. Perhaps not all transplant centers need to provide artificial lung technologies. How national health care policy would establish regional referral centers, what such centers would actually do, and how they would be structured and reimbursed would obviously be contentious. As you know, regionalization has been discussed in the past and the political wherewithal to truly study the issue has been missing. 